adiponectin. We also investigated whether these biomarkers correlated with various demographic, clinical and laboratory markers.

Methods We treated 37 RA patients with either etanercept (ETN) or certolizumab pegol (CZP) in a 12 month follow-up study. Assessments were performed at baseline, and 3, 6 and 12 months after treatment initiation. Serum chemerin and adiponectin concentrations were measured by commercially available ELISA kits (R and D System, MN and USA). PON1 and arylesterase activities were measured by spectrophotometry. In addition, age, disease duration, disease activity (DAS28), CRP, anti-CCP, IgM rheumatoid factor and plasma lipid levels were also assessed. Arterial flow-mediated vasodilation (FMD), carotid intima-media thickness (cIMT) and arterial pulse-wave velocity (PWV) were assessed by ultrasound.

Results Anti-TNF treatment resulted in a significant decrease in the levels of chemerin $(\mathrm{p}<0.001)$ and adiponectin $(\mathrm{p}<0.007)$ after 12 months. There were no significant changes in the levels of other metabolic biomarkers. We found the following correlations between the baseline values: the PON1 levels correlated with the age of patients $(R=0.466$, $\mathrm{p}=0.004)$. The adiponectin correlated with the disease activity $(\mathrm{R}=0.385, \mathrm{p}=0.030)$, HDL-C $(\mathrm{R}=0.417, \mathrm{p}=0.012)$ and the triglyceride levels $(\mathrm{R}=0.481, \mathrm{p}=0.003)$. The total cholesterol correlated with the PWV $(\mathrm{R}=0.449, \mathrm{p}=0.021)$ and the levels of the LDL-C $(\mathrm{R}=0.911, \mathrm{p}<0.001)$. The baseline triglyceride correlated with the IgM rheumatoid factor $(R=0.343$, $\mathrm{p}=0.021)$; and the levels of LDL-C correlated with the PWV values $(\mathrm{R}=0.444, \mathrm{p}=0.023)$.

Conclusions Metabolic factors, such as certain adipokines, PON1 and arylesterase may play a role in oxidative stress and atherosclerosis associated with RA. Anti-TNF treatment may affect adipokine levels.

Acknowledgements The work is supported by the GINOP2.3.2-15-2016-00015/I-KOM TEAMING project.

Disclosure of interest None declared

\section{P076 A BIOASSAY TO MEASURE TGFB ACTIVITY REVEALS DECREASED TGFB ACTIVITY IN SYSTEMIC SCLEROSIS SERUM}

${ }^{1} \mathrm{~A}$ van Caam*, ${ }^{1} \mathrm{E}$ Vitters, ${ }^{2} \mathrm{~F}$ van den Hoogen, ${ }^{2} \mathrm{M}$ Vonk, ${ }^{1} \mathrm{P}$ van der Kraan. ${ }^{1}$ Experimental Rheumatology; ${ }^{2}$ Rheumatology, Radboudumc, Nijmegen, Netherlands

\subsection{6/annrheumdis-2018-EWRR2018.93}

Introduction Systemic sclerosis (SSc) is a severe disease characterised by auto-immunity, vasculopathy and excessive fibrosis of connective tissues. The pathophysiology of SSc is still poorly understood, but its symptoms imply a role for dysregulated transforming growth factor $\beta$ (TGF $\beta$ ) signalling because this cytokine is known to regulate vascular and connective tissue biology. TGF $\beta$ circulates in blood in an inactive latent form bound to latency associated peptide and latent TGF $\beta$ binding proteins. This latent TGF $\beta$ has to be activated before it can become bioactive. With the use of a bioassay TGF $\beta$ 's bioactivity can be measured in complex mixtures like serum. This is not possible with an ELISA because this technique does not take (cellular) activation processes into account.

Objectives To determine the bioactivity of TGF $\beta$ in SSc serum compared to that of healthy control serum.

Methods Serum was collected of 10 SSc patients and 10 age and sex matched healthy controls. Primary human fibroblasts of 3 donors were transduced with $\mathrm{CAGA}_{12}$-luc which produces luciferase in response to $\mathrm{TGF} \beta / \mathrm{Smad} 3$ or BRE-luc which produces luciferase in response to $\mathrm{BMP} / \mathrm{Smad} 1 / 5$. These cells were treated with $10 \%$ serum for 16 hour and luciferase activity was measured. To activate all TGF $\beta$, sera were treated with $4 \mathrm{M} \mathrm{HCl}$ for 1 hour at RT, after which $\mathrm{pH}$ was normalised with $4 \mathrm{M} \mathrm{NaOH}$. Controls were treated with $\mathrm{HCl}$ and $\mathrm{NaOH}$ simultaneously. To verify that TGF $\beta$ signalling was measured in this reporter assay, sera were treated with antiTGF $\beta 1 / 2 / 3$ for 1 hour at RT before use.

Results Control sera significantly induced reporter activity by 4.5 -fold. However, SSc sera only induced a 2.5 -fold increase in luciferase activity, indicating significantly lower bioactivity of TGF $\beta(p<0.0001)$. This difference was not due to a difference in total TGF $\beta$ levels; after activation of all TGF $\beta$ both $\mathrm{HC}$ and SSc sera induced a similar 6-fold increase in signal strength. These data show that in HC sera approximately $75 \%$ of all TGF $\beta$ is bioactive compared to only $42 \%$ in SSc. Addition of anti-TGF $\beta 1 / 2 / 3$ inhibited all $\mathrm{CAGA}_{12}$-luc reporter activity $(\mathrm{p}<0.0001)$ of both $\mathrm{HC}$ and SSc serum, and of both acidified and not acidified sera $(p<0.0001)$, showing that our bioassay is indeed TGF $\beta$-dependent. To investigate if reduced bioactivity is a more general phenomenon we measured BMP activity. BMP proteins are structurally closely related to TGF $\beta$ and also circulate in inactive form. Both $\mathrm{HC}$ and SSc sera induced a similar 8-fold increase in BRE-luc activity, and this activity was increased to a 16 -fold induction after acidification for both groups. BMPs in SSc sera are thus not less bioactive. This illustrates the uniqueness of our observation on TGF $\beta$ bioactivity.

Conclusions TGF $\beta$ in SSc serum is less bioactive than in control serum whereas BMPs are not less bioactive.

Disclosure of interest None declared

\section{P077 EFFECTS OF IL-17 AND THE HEPATOCYTE- MONONUCLEAR CELL INTERACTIONS IN THE HEPATIC INFLAMMATORY RESPONSE}

${ }^{1} \mathrm{~A}$ Beringer*, ${ }^{1} \mathrm{ND}$ Thiam, ${ }^{2} \mathrm{~J}$ Molle, ${ }^{2} \mathrm{~B}$ Bartosch, ${ }^{1} \mathrm{P}$ Miossec. ${ }^{1} /$ mmunogenomics and Inflammation Research Unit EA4130, University of Lyon; ${ }^{2}$ Inserm U1052, Cancer Research Centre, Lyon, France

\subsection{6/annrheumdis-2018-EWRR2018.94}

Introduction Interleukin-17A (IL-17), tumour necrosis factor- $\alpha$ $(\mathrm{TNF} \alpha)$ and IL-6 are pro-inflammatory cytokines involved in many autoimmune and inflammatory diseases including rheumatoid arthritis. Because most acute-phase proteins are produced by the liver, the hepatic response plays an important role in systemic inflammation. By producing chemokines such as CCL-20 that attract Th17 cells, hepatocytes can recruit mononuclear cells in liver, which leads to chronic hepatitis. Objectives To determine the effect of the IL-17/TNF $\alpha$ combination and the contribution of hepatocyte-peripheral blood mononuclear cell (PBMC) interactions in the hepatic inflammatory response.

Methods Human HepaRG cell line and primary human hepatocytes $(\mathrm{PHH})$ were cultured with or without IL-6, anti-IL-6 receptor (anti-IL-6R) antibody, IL-17 and/or TNF $\alpha$. For co-cultures, PBMC from healthy donors activated or not with phytohemagglutinin (PHA) were added on hepatocytes at a 5:1 ratio. Transwell system was used to study the contribution of the direct cell-cell contact. Cytokine expression and production were quantified by qRT-PCR and ELISA respectively and 
the secretion of C-reactive protein (CRP) by an automated analyzer.

Results IL-17 and TNF $\alpha$ induced in synergy the hepatic IL-6 production $(>8$ fold, $\mathrm{p}<0.01)$ and CCL-20 expression ( $>100$ fold, $\mathrm{p}<0.01$ ). CRP secretion was induced by IL-17/ TNF $\alpha$ and the anti-IL-6R inhibited this induction. CCL-20 expression was not increased by IL-6 stimulation or reduced by the addition of the anti-IL-6R to the IL-17/TNF $\alpha$ treatment. In HepaRG cell-PBMC cultures, IL-6 and IL-17 were produced only in PHA-activated conditions and the IL-6 and IL-17 levels were higher in co-cultures vs PBMC monocultures $(>14$ and $>2$ fold respectively, $\mathrm{p}<0.01)$. Transwell system that avoids direct cell-cell contact decreased the IL-6 and IL17 secretion by 4- and 2-fold respectively in PHA-activated HepaRG cell-PBMC cultures. CRP production increased when $\mathrm{PHH}$ were cultured in presence of PHA-activated PBMC compared to $\mathrm{PHH}$ alone with PHA.

Conclusions The IL-17/TNF $\alpha$ synergistic effect on hepatocytes mediates systemic inflammation by inducing CRP secretion through the IL-6 pathway and mononuclear cell recruitment by acting on CCL-20 expression in an IL- 6 independent manner. Direct and indirect hepatocyte-PBMC interactions contribute to the hepatic inflammatory response by increasing the IL6 and IL-17 secretion. The induction of Th17 cell differentiation by IL- 6 and the increase of CCL- 20 mediated Th17 cell recruitment by IL-17 may lead to a vicious and chronic inflammatory cycle.

Disclosure of interest None declared

\section{P078 A GENETIC VARIANT OF IL-32 IS ASSOCIATED WITH THE EX VIVO CYTOKINE PRODUCTION OF ANTI-TNF TREATED PBMCS ISOLATED FROM RHEUMATOID ARTHRITIS PATIENTS}

\footnotetext{
${ }^{1,2} \mathrm{C}$ Popa* ${ }^{3} \mathrm{M}$ Damen, ${ }^{3} \mathrm{~K}$ Schraa, ${ }^{2} \mathrm{~L}$ Tweehuysen, ${ }^{2} \mathrm{~A}$ den Broeder, ${ }^{3} \mathrm{M}$ Netea, ${ }^{3} \mathrm{~L}$ Joosten. ${ }^{1}$ Rheumatology, Radboud UMC; ${ }^{2}$ Rheumatology, Sint Maartenskliniek; ${ }^{3}$ Internal Medicine, Radboud UMC, Nijmegen, Netherlands
}

\subsection{6/annrheumdis-2018-EWRR2018.95}

Introduction Since the introduction of biologics in the treatment of rheumatoid arthritis (RA) disease outcome improved. Still, about $40 \%$ of RA patients do not respond to therapy with TNF $\alpha$ blockers. Previously, a strong link between TNF $\alpha$ and interleukin (IL) -32 has been reported in RA. ${ }^{1}$

Objectives We hypothesise that a promoter single nucleotide polymorphism (SNP) in IL-32 can affect clinical responsiveness to anti-TNF $\alpha$ treatment in RA patients, functioning as a new biomarker in treatment of RA. ${ }^{2}$

Methods Peripheral mononuclear cells (PBMCs) from RA patients and healthy individuals were stimulated with RPMI or recombinant human (rh)TNF $\alpha$ to study the mRNA and protein expression of IL-32 and other pro-inflammatory cytokines. Moreover, disease activity scores (DAS28), 'in vitro response' and clinical response to anti-TNF $\alpha$ therapy (etanercept, adalimumab), of RA patients were measured and all were stratified for the IL-32 SNP (C/T).

Results Stimulation of PBMCs from RA patients was followed by higher IL-32 protein production and a tendency towards higher IL-32 $\beta$ and IL-32 $\gamma$ mRNA expression compared to healthy individuals. When data was stratified for the IL-32 promoter SNP, patients bearing the CC genotype showed higher IL-32 protein expression. Of interest, these patients also produced more cytokines. Even though the DAS28 did not depend on the presence of the promoter SNP, the 'ex vivo' cytokine response did have a different pattern in clinical responders depending on the genotype.

Conclusions IL-32 mRNA and protein production was higher in RA patients compared to healthy individuals, with a trend towards higher concentrations in patients bearing the CC genotype. Regardless of the fact that the promoter SNP was not associated with disease activity, IL-1 beta production in the CC-genotype might predict clinical response to either etanercept or adalimumab.

\section{REFERENCES}

1. Heinhuis B, et al. Ann Rheum Dis 2011;70:660-7.

2. Damen MS, et al. Sci Rep 2017;7:41629.

Disclosure of interest None declared

\section{P079 INTERLEUKIN-27 REGULATES THE MAGNITUDE OF THE ECTOPIC GERMINAL CENTRE RESPONSE IN A VIRAL- INDUCIBLE MODEL OF SIALADENITIS}

${ }^{1} \mathrm{D}$ Lucchesi*, ${ }^{1} \mathrm{E}$ Pontarini, ${ }^{1} \mathrm{R}$ Coleby, ${ }^{2} \mathrm{GW}$ Jones, ${ }^{2} \mathrm{DG}$ Hill, ${ }^{1} \mathrm{C}$ Pitzalis, ${ }^{1} \mathrm{M}$ Bombardieri. ${ }^{1}$ Experimental Medicine and Rheumatology, Queen Mary University of London, London; ${ }^{2}$ Division of Infection and Immunity, School of Medicine, Cardiff University, Cardiff, UK

\subsection{6/annrheumdis-2018-EWRR2018.96}

Introduction Ectopic lymphoid structures (ELS) are leukocytes aggregates that form in tissues affected by chronic inflammation. In autoimmunity, ELS play an active role in disease progression and are typically associated with aggressive disease. Thus, understanding the mechanisms that trigger ELS formation is of paramount importance for therapeutic targeting of this process. Interleukin-27 (IL-27) is prominently associated with the negative control of adaptive immunity, and in particular in the suppression of Th17-type responses.

Objectives To elucidate the role of IL-27 in the control of lymphoid neogenesis and its functional relationship with aberrant IL-17 production in a murine model of inducible ELS, where the local administration of a replication-deficient adenovirus (AdV) triggers the formation of ectopic germinal centres in salivary glands (SG).

Methods A single administration of AdV was delivered by cannulation directly into the SG of wild-type (WT) and IL-27Rdeficient $\left(I l 27 \mathrm{ra}^{-/}\right)$mice. For IL-17A blockade, an anti-mouse IL-17A antibody or IgG control was administered systemically. ELS development and peripheral immune responses were temporally tracked by immuno-histopathology, flow cytometry, and real-time qPCR.

Results AdV cannulation induced an early upregulation of IL27 and IL-27R in WT mice SG, which was mirrored by an increase in the infiltration of IL-27-producing T, B and NK cells. AdV-challenged $\mathrm{Il}_{2} 7 \mathrm{ra}^{-/-}$mice developed exacerbated salivary gland inflammation, and by day- 19 post $\mathrm{AdV}$ challenge developed larger and more abundant ELS as compared to WT mice. Moreover, $\mathrm{Il}_{2} 7 \mathrm{ra}^{-/}$mice displayed a heightened expression of homeostatic cytokines, chemokines and their corresponding receptors that are required for lymphoid neogenesis (e.g., Cxcl13, Ccl19 and Ltb). IL-27R-deficient mice also displayed elevated markers of functional germinal centre responses (e.g., activation-induced deaminase, AID). Underpinning the exaggerated development of ELS in $\mathrm{Il}_{2} 7 \mathrm{ra}^{-/-}$mice was the preferential expansion of IL-17-producing $\mathrm{T}$ helper (Th)17 cells, which was linked to a reduction in the Th1 cell 